ГORIGINAL ARTICLE

Volume 9 Issue 22017

DOI: 10.21315/eimj2017.9.2.1

ARTICLE INFO

Submitted: 26-01-2017

Accepted: 08-03-2017

Online: 30-06-2017

\section{Development and Validation of the Knowledge and Clinical Reasoning of Acute Asthma Management in Emergency Department (K-CRAMED)}

\author{
Mohd Muzammil Ozair', Kamarul Aryffin Baharuddin², Saiful Azlan \\ Mohamed $^{2}$, Wafaak Esa ${ }^{3}$, Muhamad Saiful Bahri Yusoff ${ }^{4}$ \\ ${ }^{1}$ Emergency Department, Hospital Angkatan Tentera Wilayah Kota \\ Kinabalu, Pangkalan TLDM Kota Kinabalu, Sabah, MALAYSIA \\ ${ }^{2}$ Emergency Department, \\ ${ }^{3}$ Department of Community Medicine, \\ ${ }^{4}$ Department of Medical Education, School of Medical Science, \\ Universiti Sains Malaysia, MALAYSIA
}

To cite this article: Ozair MM, Baharuddin KA, Mohamed SA, Esa W, Yusoff MSB. Development and validation of the knowledge and clinical reasoning of acute asthma management in emergency department (K-CRAMED). Education in Medicine Journal. 2017;9(2):1-17. https://doi.org/10.21315/ eimj2017.9.2.1

To link to this article: https://doi.org/10.21315/eimj2017.9.2.1

\title{
ABSTRACT
}

Introduction: Suboptimal management of asthma can lead to increase morbidity and mortality. Unfortunately, this has become global issue and approximately $40 \%$ of asthmatic patients received suboptimal management in emergency department. Therefore, this study aimed to develop a tool to assess knowledge and clinical reasoning of healthcare providers on acute asthmatic management in emergency setting. Method: The tool was developed via three phases: (a) domain identification, (b) domain blueprinting based the Global Initiative of Asthma (GINA) and the British Thoracic Society (BTS) guidelines, and (c) item generation for each domain for assessing knowledge and clinical reasoning. Three forms of validity evidence related to content, response process and internal structure were appraised. Content validity index (CVI), face validity index (FVI), and intraclass correlation coefficient (ICC) estimate the content validity, response process and internal structure of the tool. Results: A new tool was developed, named as Knowledge and Clinical Reasoning of Acute Asthma Management in Emergency Department (K-CRAMED), which assesses knowledge and clinical reasoning on three domains related to management of acute asthma - diagnosis, treatment and disposition. CVI values for the three domains were more than 0.83 . FVI values for the three domains among doctors and paramedics were at least 0.83 . The ICC between scores given by emergency specialists was 0.989 (CI 95\% 0.982, 0.994, $p$-value < 0.001). Conclusion: The newly developed tool, named as K-CRAMED, is a valid tool to assess knowledge and clinical reasoning of healthcare providers who manage patients with acute asthma. Further validation is required to verify its validity in other setting.

Keywords: Asthma management, Clinical reasoning, Content validity, Face validity, Knowledge 


\section{INTRODUCTION}

Asthma is one of the commonest noncommunicable and chronic respiratory illnesses. It affects all age groups and often started during childhood. It is characterised by recurrent episode of shortness of breath, chest tightness, cough and wheezing. The exacerbation of asthma varies in frequency and severity from individual to individual. In affected individuals, symptoms might occur several times in a day or week and others may become worse during physical activity, at night or in cold environment (1).

World Health Organization (WHO) estimates about 235 millions of asthma patients worldwide, predominantly children (2). Asthma is public health issues in all countries regardless of development level. In addition, more than $80 \%$ of asthma deaths were reported in a group of low income countries (3). WHO also reported that suboptimal management of asthma will burden not only to individuals and communities, it will possibly restricting daily activities of affected individuals for lifetime (4).

All government medical facilities in Malaysia provide service for acute asthma relieve whether in primary, secondary or tertiary centre. Besides, most of the hospitals with a proper emergency department (ED) set up have specific asthma bays to facilitate the early treatment of asthmatic attack patients. Thus, all of these patients get the fast track medical treatment once they are diagnosed of asthma exacerbation.

From the local and unpublished record, $33 \%$ of patients did not receive oral corticosteroid and they revisited ED within 48 hours following asthmatic attack, 57\% patients received delayed asthma treatment due to non-identified symptoms during the triage process, and $50 \%$ patients received non-standard dose of asthma medication during the visit. This represents $40 \%$ of asthmatic patients received treatment that did not comply with the recommended guidelines - suboptimal management.
Several literatures reported that some of the centres face similar problem of non-adherence to the guidelines by the healthcare provider (HCP).

Ting mentioned that many studies found that HCP generally did not use and adhere to the guidelines (5). He also demonstrated four common physician reasons for poor adherence to guidelines that were,

1. Could not remember classification parameters of asthma severity,

2. Could not remember various brand and exact dosages of inhaled steroids for different asthma severity,

3. Could not remember to ask about various triggers of asthma, and

4. Did not have sufficient time or resources to provide asthma education and an asthma action plan upon discharge.

A study in Kuwait also found physicians at primary health care centres had a low adherence rate to asthma guidelines. Although they had high positive attitude toward asthma yet their knowledge and practice require improvement (6). This is also supported by two studies conducted in the middle east and European countries (7, 8) that found HCP poorly comply with the recommended asthma guideline and asthma management. An Asian study also showed poor adherence to asthma guideline in several parts of recommendation (9). These facts suggest that suboptimal management of asthmatic patients is a global issue.

Due to the aforementioned issues, this study aimed to develop a valid tool for assessing healthcare providers' knowledge and clinical reasoning on the management of asthma patients during acute exacerbation. In general, there are five sources of primary validity evidence outlined in the literature, which are content, response process, internal structure, relation to other variables and consequences of a measurement (10). In this study, only three validity evidence were investigated that were content, response process and internal structure. 


\section{METHODOLOGY}

Figure 1 summarised the methodology for developing and validating a new tool, named as K-CRAMED, to assess knowledge and clinical reasoning of acute asthma management among healthcare providers. The details of each phase were elaborated in the subsequent subsections.

\section{Development Phase}

Based on the clinical observation, two main components (i.e., knowledge and clinical reasoning) were identified, and each component consisted of three domains (i.e., diagnosis, treatment and disposition). A supplied type question assessed knowledge whereby candidates provide answer to the question (11). Clinical reasoning was assessed by the script concordance test (SCT) (12), in which candidates provide answer to a range of clinical scenario from disagree to agree based on a Likert scale and a panel of expert set the SCT scoring rubric. Diagnosis domain focused on recognising the symptoms and signs, the necessary investigation and the severity. Treatment domain focused on the choice and dosage of medications, side effect of common medications and preparation of common medications. Disposition domain focused on the decision for inpatient treatment in ED or outpatient treatment with discharge management plan.

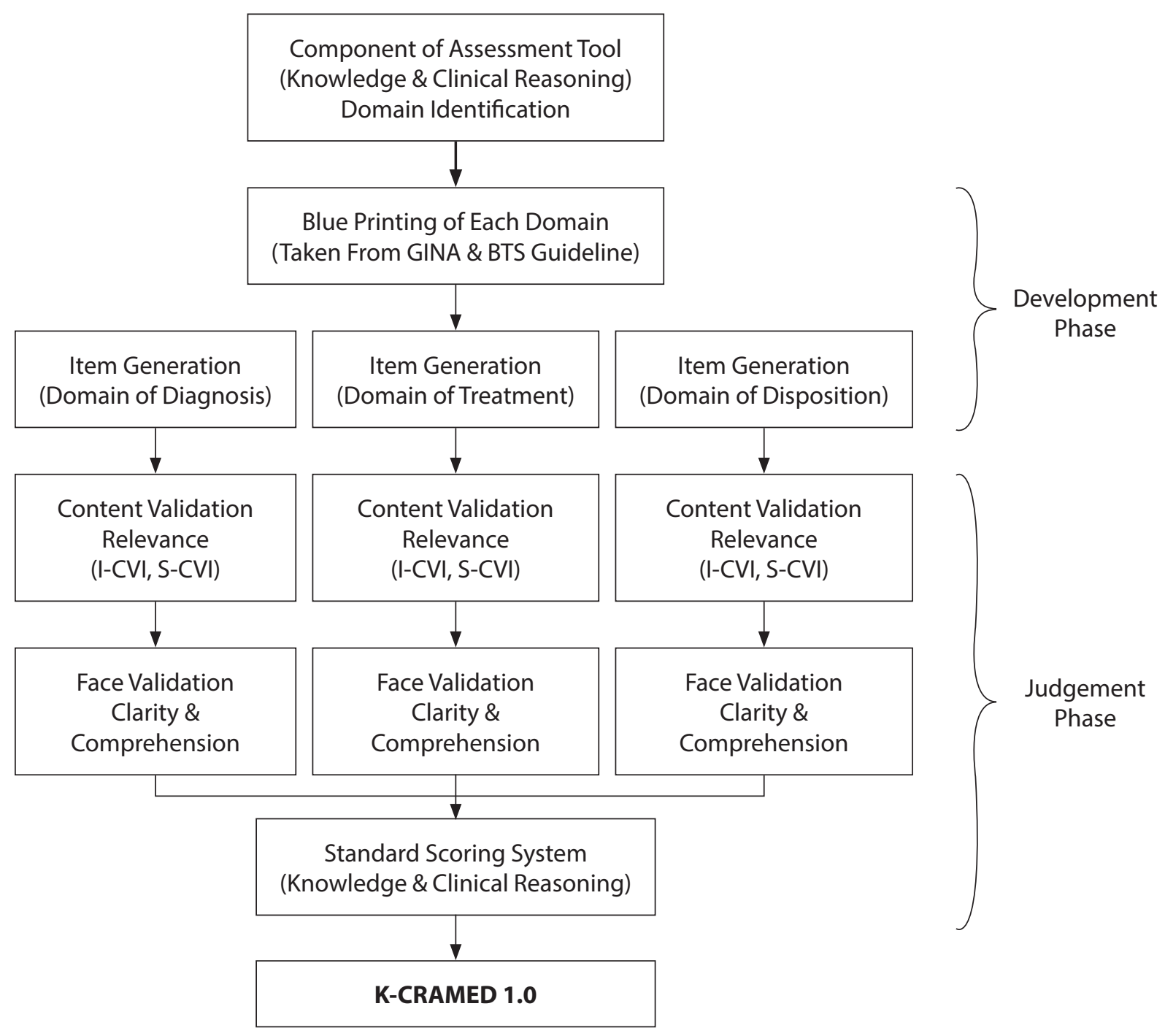

Figure 1: The flowchart of development and validation of K-CRAMED. 
A blueprint of each domain were developed based on two main guidelines which that were the Global Initiative of Asthma (GINA) and the British Thoracic Society (BTS) $(13,14)$. Potential items of each domain in each component were generated based on the blueprint via Delphi Technique
(15). Four meetings were arranged with a panel of expert and final agreement was achieved after rewording, rephrasing, and adjustment to prevent from bias and ambiguous meaning of each item. The objectives of the components and domains were summarised in Table 1 to Table 3.

Table 1: Final objectives for items of diagnosis domain by two components

\begin{tabular}{|c|c|c|}
\hline Item & Objective & Component \\
\hline $\mathrm{K} 1.1$ & To assess the knowledge of asthma symptoms in asthma definition & \multirow{5}{*}{ Knowledge } \\
\hline $\mathrm{K} 1.2$ & To assess the knowledge of commonest symptom in asthma exacerbation & \\
\hline K1.3 & To assess the knowledge of investigating allergy status & \\
\hline K1.4 & To assess the knowledge of common asthma presentation in paediatric age group & \\
\hline K1.5 & To assess the knowledge of extreme manifestation of asthma exacerbation & \\
\hline CR1 & To challenge the diagnosis of asthma in a stridor patient & \multirow{7}{*}{$\begin{array}{l}\text { Clinical } \\
\text { reasoning }\end{array}$} \\
\hline CR2 & To challenge the diagnosis of asthma in a first time wheezed patient & \\
\hline CR3 & To challenge the diagnosis of asthma in wheezed paediatric patient & \\
\hline CR4 & To challenge the diagnosis of anaphylaxis in an eczema patient & \\
\hline CR5 & To challenge the decision to proceed with chest radiograph in asthma patient & \\
\hline CR6 & $\begin{array}{l}\text { To challenge the decision to proceed with arterial blood gases in normal oxygen } \\
\text { saturation patient }\end{array}$ & \\
\hline CR7 & $\begin{array}{l}\text { To challenge the decision to proceed with peak expiratory flow rate in a distress } \\
\text { patient }\end{array}$ & \\
\hline
\end{tabular}

Table 2: Final objectives for items of treatment domain by two components

\begin{tabular}{|c|c|c|}
\hline Item & Objective & Component \\
\hline K2.1 & To assess the knowledge of Ipratropium Bromide frequency & \multirow{8}{*}{ Knowledge } \\
\hline $\mathrm{K} 2.2$ & To assess the knowledge of Salbutamol dosage in adult patient & \\
\hline $\mathrm{K} 2.3$ & To assess the knowledge of medication to use in extreme manifestation of asthma & \\
\hline $\mathrm{K} 2.4$ & To assess the knowledge of "how to prepare" Salbutamol nebulisation & \\
\hline K2.5 & To assess the knowledge of Combivent preparation in Malaysia & \\
\hline K2.6 & $\begin{array}{l}\text { To assess the knowledge of recommended route of administration for Salbutamol } \\
\text { in paediatric age group }\end{array}$ & \\
\hline K2.7 & To assess the knowledge of usage of Hydrocortisone & \\
\hline $\mathrm{K} 2.8$ & $\begin{array}{l}\text { To assess the knowledge of "how to prepare" mixed Salbutamol and Ipratropium } \\
\text { Bromide nebulization in adult patient }\end{array}$ & \\
\hline CR8 & To challenge the decision to give Hydrocortisone in a persistent vomiting patient & \multirow{5}{*}{$\begin{array}{l}\text { Clinical } \\
\text { reasoning }\end{array}$} \\
\hline CR9 & To challenge the decision to give Magnesium Sulphate in a hypotensive patient & \\
\hline CR10 & To challenge the decision to give intravenous Salbutamol in a tachycardia patient & \\
\hline CR11 & To challenge the decision to give repeated Ipratropium Bromide within one hour & \\
\hline CR12 & $\begin{array}{l}\text { To challenge the decision to use Ketamine in the absent of ventilator machine } \\
\text { support }\end{array}$ & \\
\hline
\end{tabular}


Table 3: Final objectives for items of disposition domain by two components

\begin{tabular}{|c|c|c|}
\hline Item & Objective & Component \\
\hline K3.1 & To assess the knowledge of prescribing steroid as discharge asthma medication & Knowledge \\
\hline $\mathrm{K} 3.2$ & To assess the knowledge of asthma related illness and proper follow up care & \\
\hline K3.3 & To assess the knowledge of medication to avoid in asthma patient & \\
\hline K3.4 & $\begin{array}{l}\text { To assess the knowledge of safe criteria for outpatient decision in a patient } \\
\text { recovered from asthma exacerbation }\end{array}$ & \\
\hline K3.5 & $\begin{array}{l}\text { To assess the knowledge of prescribing Ipratropium Bromide as discharge asthma } \\
\text { medication }\end{array}$ & \\
\hline K3.6 & To assess the knowledge of asthma discharge plan apart of medication & \\
\hline CR13 & $\begin{array}{l}\text { To challenge the decision to discharge a recovered patient that received multiple } \\
\text { types of asthma medication during exacerbation }\end{array}$ & $\begin{array}{l}\text { Clinical } \\
\text { reasoning }\end{array}$ \\
\hline CR14 & $\begin{array}{l}\text { To challenge the decision of giving oral steroid medication in a patient that } \\
\text { received inhaler steroid medication }\end{array}$ & \\
\hline CR15 & $\begin{array}{l}\text { To challenge the decision to admit a persistent wheezing patient that fulfil other } \\
\text { criteria of home discharge }\end{array}$ & \\
\hline
\end{tabular}

\section{Judgement Phase}

Each item underwent content validation (CVI), face validation (FVI), inter-rater reliability and standard setting scoring. CVI assessed relevance and representativeness of each item to a specific domain by the panel of expert. Ten panels were recruited from lecturers in the ED and they rated each item based on a Likert scale ranging from 0 (i.e., not relevant or not represent) to 4 (i.e., highly relevant or highly represent). Items were refined after the first meeting based on the panel recommendation. At the final meeting, the panel raw rating were gathered and entered into Microsoft Excel. The calculation of item-level content validity index (I-CVI); scale-level content validity index (S-CVI); scale-level content validity index, universal agreement calculation method (S-CVI/UA); and scale-level content validity index, averaging calculation method (S-CVI/Ave); were estimated manually. Beck et al. recommended that each item rated as 3 or 4 were converted to valid ('1') and item rated as 0,1 or 2 were converted to nonvalid ('0') (16). Individual item assessment and average of each item will take into consideration during the data presentation. The additional universal agreement would facilitate the assessment of inter-rater reliability.
S-CVI/Ave was calculated by two formulas as follow:

$$
\begin{aligned}
& \mathrm{I}-\mathrm{CVI}=\underset{\text { (agreed item) }}{\text { rater })} \\
& \mathrm{S}-\mathrm{CVI} / \text { (number of } \\
& \quad \begin{array}{l}
\text { (summation all I-CVI) } / \\
\text { (number of item) }
\end{array}
\end{aligned}
$$

The first method was to get all I-CVI value and divide it by the number of items. The second method was to get the average proportion of each rater. Then, S-CVI/ UA was calculated by getting the number of items which had 100\% agreement, and divided by the total number of items in that specific domain (17). A new tool should achieve at least $80 \%$ or higher agreement to be considered as acceptable content validity (18).

FVI assessed comprehensibility and clarity of each item by respondents. Paramedics (i.e., medical assistants and nurses) and doctors who worked in the ED were invited and 30 respondents agreed to participate. The items were rated based on a Likert scale ranging from 0 (i.e., difficult clarity or difficult comprehensibility) to 4 (i.e., easy clarity or easy comprehensibility). The raw scores were entered in Microsoft Excel and calculated for the item-level face validity index (I-FVI) for each comprehensibility 
and clarity. Formula calculated for FVI as follow:

$$
\begin{aligned}
\mathrm{FVI}= & \text { (summation of FVI score) } \\
& /(\max \text { score } * \text { number of } \\
& \text { rater })
\end{aligned}
$$

Data was also entered into SPSS 22 to estimate the inter-rater reliability and standard setting scoring was set by a panel of experts. The standard scoring for the knowledge component was achieved by a close meeting with a panel of expert - they was not involved in the judgement phase. The panel was briefed regarding the need of scoring system and each item was examined item-by-item before the final decision.

The standard scoring was also set for the clinical reasoning component. The principle applied for this component was to check the agreement between experts by a script concordance test. One meeting was arranged with a panel of expert (i.e., 10 lecturers in an emergency department, and scores of each item was calculated based on their agreement.
Ethical approval was obtained from the Ethical Committee, Universiti Sains Malaysia USM/JEPeM/283.3(1.6).

\section{RESULTS}

\section{Content Validity Index (CVI)}

In the domain of diagnosis (Table 4), item K1.3 was removed based on the expert panel's recommendation - the question was not relevant as evident by I-CVI value was zero. A total 10 out of 12 items achieved acceptable universal agreement between experts $(\mathrm{S}-\mathrm{CVI} / \mathrm{UA}=0.83)$.

CVI for the treatment domain (Table 5), items K2.5 and CR10 based on the expert panel's recommendation - the question was not relevant as evident by I-CVI value was zero. A total 11 out of 13 items achieved acceptable universal agreement between

\begin{tabular}{|c|c|c|c|c|c|c|c|c|c|c|c|c|}
\hline & $\begin{array}{c}\text { Rater } \\
1\end{array}$ & $\begin{array}{c}\text { Rater } \\
\mathbf{2}\end{array}$ & $\begin{array}{c}\text { Rater } \\
\mathbf{3}\end{array}$ & $\begin{array}{c}\text { Rater } \\
4\end{array}$ & $\begin{array}{c}\text { Rater } \\
5\end{array}$ & $\begin{array}{c}\text { Rater } \\
6\end{array}$ & $\begin{array}{c}\text { Rater } \\
7\end{array}$ & $\begin{array}{c}\text { Rater } \\
8\end{array}$ & $\begin{array}{c}\text { Rater } \\
9\end{array}$ & $\begin{array}{c}\text { Rater } \\
10\end{array}$ & $\begin{array}{l}\text { Experts in } \\
\text { agreement }\end{array}$ & $\begin{array}{l}\text { Items } \\
\text { CVI }\end{array}$ \\
\hline \multicolumn{13}{|l|}{ Knowledge } \\
\hline K1.1 & $\sqrt{ }$ & $\sqrt{ }$ & $\sqrt{ }$ & $\sqrt{ }$ & $\sqrt{ }$ & $\sqrt{ }$ & $\sqrt{ }$ & $\sqrt{ }$ & $\sqrt{ }$ & $\sqrt{ }$ & 10 & 1.00 \\
\hline $\mathrm{K} 1.2$ & $\sqrt{ }$ & - & $\sqrt{ }$ & $\sqrt{ }$ & $\sqrt{ }$ & $\sqrt{ }$ & $\sqrt{ }$ & $\sqrt{ }$ & $\sqrt{ }$ & $\sqrt{ }$ & 9 & 0.90 \\
\hline K1.3 & - & - & - & - & - & - & - & - & - & - & 0 & 0.00 \\
\hline K1.4 & $\sqrt{ }$ & $\sqrt{ }$ & $\sqrt{ }$ & $\sqrt{ }$ & $\sqrt{ }$ & $\sqrt{ }$ & $\sqrt{ }$ & $\sqrt{ }$ & $\sqrt{ }$ & $\sqrt{ }$ & 10 & 1.00 \\
\hline K1.5 & $\sqrt{ }$ & $\sqrt{ }$ & $\sqrt{ }$ & $\sqrt{ }$ & $\sqrt{ }$ & $\sqrt{ }$ & $\sqrt{ }$ & $\sqrt{ }$ & $\sqrt{ }$ & $\sqrt{ }$ & 10 & 1.00 \\
\hline Proportion & 0.80 & 0.60 & 0.80 & 0.80 & 0.80 & 0.80 & 0.80 & 0.80 & 0.80 & 0.80 & & \\
\hline \multicolumn{13}{|l|}{$\begin{array}{l}\text { Clinical } \\
\text { reasoning }\end{array}$} \\
\hline CR1 & $\sqrt{ }$ & $\sqrt{ }$ & $\sqrt{ }$ & $\sqrt{ }$ & $\sqrt{ }$ & $\sqrt{ }$ & $\sqrt{ }$ & $\sqrt{ }$ & $\sqrt{ }$ & $\sqrt{ }$ & 10 & 1.00 \\
\hline CR2 & $\sqrt{ }$ & $\sqrt{ }$ & $\sqrt{ }$ & $\sqrt{ }$ & $\sqrt{ }$ & $\sqrt{ }$ & $\sqrt{ }$ & $\sqrt{ }$ & $\sqrt{ }$ & $\sqrt{ }$ & 10 & 1.00 \\
\hline CR3 & $\sqrt{ }$ & $\sqrt{ }$ & $\sqrt{ }$ & $\sqrt{ }$ & $\sqrt{ }$ & $\sqrt{ }$ & $\sqrt{ }$ & $\sqrt{ }$ & $\sqrt{ }$ & $\sqrt{ }$ & 10 & 1.00 \\
\hline CR4 & $\sqrt{ }$ & $\sqrt{ }$ & $\sqrt{ }$ & $\sqrt{ }$ & $\sqrt{ }$ & $\sqrt{ }$ & $\sqrt{ }$ & $\sqrt{ }$ & $\sqrt{ }$ & $\sqrt{ }$ & 10 & 1.00 \\
\hline CR5 & $\sqrt{ }$ & $\sqrt{ }$ & $\sqrt{ }$ & $\sqrt{ }$ & $\sqrt{ }$ & $\sqrt{ }$ & $\sqrt{ }$ & $\sqrt{ }$ & $\sqrt{ }$ & $\sqrt{ }$ & 10 & 1.00 \\
\hline CR6 & $\sqrt{ }$ & $\sqrt{ }$ & $\sqrt{ }$ & $\sqrt{ }$ & $\sqrt{ }$ & $\sqrt{ }$ & $\sqrt{ }$ & $\sqrt{ }$ & $\sqrt{ }$ & $\sqrt{ }$ & 10 & 1.00 \\
\hline \multirow[t]{2}{*}{ CR7 } & $\sqrt{ }$ & $\sqrt{ }$ & $\sqrt{ }$ & $\sqrt{ }$ & $\sqrt{ }$ & $\sqrt{ }$ & $\sqrt{ }$ & $\sqrt{ }$ & $\sqrt{ }$ & $\sqrt{ }$ & 10 & 1.00 \\
\hline & & & & & & & & & & & S-CVI/Ave & 0.91 \\
\hline Proportion & 1.00 & 1.00 & 1.00 & 1.00 & 1.00 & 1.00 & 1.00 & 1.00 & 1.00 & 1.00 & & \\
\hline
\end{tabular}
experts $(\mathrm{S}-\mathrm{CVI} / \mathrm{UA}=0.85)$

Table 4: Ratings on knowledge and clinical reasoning (diagnosis domain) scale by 10 experts

Note. I-CVI, item-level content validity index; scale-level content validity index, universal agreement method $(\mathrm{S}-\mathrm{CVI} / \mathrm{UA})=.83$; scale-level content validity index, averaging method $(\mathrm{S}-\mathrm{CVI} / \mathrm{Ave})=.91$; average proportion of items judged relevant across the ten experts $=.91$. 
Table 5: Ratings on knowledge and clinical reasoning (treatment domain) scale by 10 experts

\begin{tabular}{|c|c|c|c|c|c|c|c|c|c|c|c|c|}
\hline & $\begin{array}{c}\text { Rater } \\
1\end{array}$ & $\begin{array}{c}\text { Rater } \\
2\end{array}$ & $\begin{array}{c}\text { Rater } \\
\mathbf{3}\end{array}$ & $\begin{array}{c}\text { Rater } \\
4\end{array}$ & $\begin{array}{c}\text { Rater } \\
5\end{array}$ & $\begin{array}{c}\text { Rater } \\
6\end{array}$ & $\begin{array}{c}\text { Rater } \\
7\end{array}$ & $\begin{array}{c}\text { Rater } \\
\mathbf{8}\end{array}$ & $\begin{array}{c}\text { Rater } \\
9\end{array}$ & $\begin{array}{c}\text { Rater } \\
10\end{array}$ & $\begin{array}{l}\text { Experts in } \\
\text { agreement }\end{array}$ & $\begin{array}{l}\text { Items } \\
\text { CVI }\end{array}$ \\
\hline \multicolumn{13}{|l|}{ Knowledge } \\
\hline K2.1 & $\sqrt{ }$ & $\sqrt{ }$ & $\sqrt{ }$ & $\sqrt{ }$ & $\sqrt{ }$ & $\sqrt{ }$ & $\sqrt{ }$ & $\sqrt{ }$ & $\sqrt{ }$ & $\sqrt{ }$ & 10 & 1.00 \\
\hline K2.2 & $\sqrt{ }$ & $\sqrt{ }$ & $\sqrt{ }$ & $\sqrt{ }$ & $\sqrt{ }$ & $\sqrt{ }$ & $\sqrt{ }$ & $\sqrt{ }$ & $\sqrt{ }$ & $\sqrt{ }$ & 10 & 1.00 \\
\hline K2.3 & $\sqrt{ }$ & $\sqrt{ }$ & $\sqrt{ }$ & $\sqrt{ }$ & $\sqrt{ }$ & $\sqrt{ }$ & $\sqrt{ }$ & $\sqrt{ }$ & $\sqrt{ }$ & $\sqrt{ }$ & 10 & 1.00 \\
\hline K2.4 & $\sqrt{ }$ & $\sqrt{ }$ & $\sqrt{ }$ & $\sqrt{ }$ & $\sqrt{ }$ & $\sqrt{ }$ & $\sqrt{ }$ & $\sqrt{ }$ & $\sqrt{ }$ & $\sqrt{ }$ & 10 & 1.00 \\
\hline K2.5 & - & - & - & - & - & - & - & - & - & - & 0 & 0.00 \\
\hline K2.6 & $\sqrt{ }$ & $\sqrt{ }$ & $\sqrt{ }$ & $\sqrt{ }$ & $\sqrt{ }$ & $\sqrt{ }$ & $\sqrt{ }$ & $\sqrt{ }$ & $\sqrt{ }$ & $\sqrt{ }$ & 10 & 1.00 \\
\hline K2.7 & $\sqrt{ }$ & $\sqrt{ }$ & $\sqrt{ }$ & $\sqrt{ }$ & $\sqrt{ }$ & $\sqrt{ }$ & $\sqrt{ }$ & $\sqrt{ }$ & $\sqrt{ }$ & $\sqrt{ }$ & 10 & 1.00 \\
\hline K2.8 & $\sqrt{ }$ & $\sqrt{ }$ & $\sqrt{ }$ & $\sqrt{ }$ & $\sqrt{ }$ & $\sqrt{ }$ & $\sqrt{ }$ & $\sqrt{ }$ & $\sqrt{ }$ & $\sqrt{ }$ & 10 & 1.00 \\
\hline Proportion & 0.88 & 0.88 & 0.88 & 0.88 & 0.88 & 0.88 & 0.88 & 0.88 & 0.88 & 0.88 & & \\
\hline \multicolumn{13}{|l|}{$\begin{array}{l}\text { Clinical } \\
\text { reasoning }\end{array}$} \\
\hline CR8 & $\sqrt{ }$ & $\sqrt{ }$ & $\sqrt{ }$ & $\sqrt{ }$ & $\sqrt{ }$ & $\sqrt{ }$ & $\sqrt{ }$ & $\sqrt{ }$ & $\sqrt{ }$ & $\sqrt{ }$ & 10 & 1.00 \\
\hline CR9 & $\sqrt{ }$ & $\sqrt{ }$ & $\sqrt{ }$ & $\sqrt{ }$ & $\sqrt{ }$ & $\sqrt{ }$ & $\sqrt{ }$ & $\sqrt{ }$ & $\sqrt{ }$ & $\sqrt{ }$ & 10 & 1.00 \\
\hline CR10 & - & - & - & - & - & - & - & - & - & - & 0 & 0.00 \\
\hline CR11 & $\sqrt{ }$ & $\sqrt{ }$ & $\sqrt{ }$ & $\sqrt{ }$ & $\sqrt{ }$ & $\sqrt{ }$ & $\sqrt{ }$ & $\sqrt{ }$ & $\sqrt{ }$ & $\sqrt{ }$ & 10 & 1.00 \\
\hline \multirow[t]{2}{*}{ CR12 } & $\sqrt{ }$ & $\sqrt{ }$ & $\sqrt{ }$ & $\sqrt{ }$ & $\sqrt{ }$ & $\sqrt{ }$ & $\sqrt{ }$ & $\sqrt{ }$ & $\sqrt{ }$ & $\sqrt{ }$ & 10 & 1.00 \\
\hline & & & & & & & & & & & S-CVI/Ave & 0.85 \\
\hline Proportion & 0.80 & 0.80 & 0.80 & 0.80 & 0.80 & 0.80 & 0.80 & 0.80 & 0.80 & 0.80 & & \\
\hline
\end{tabular}

Note. I-CVI, item-level content validity index; scale-level content validity index, universal agreement method (S-CVI/UA) $=.85$; scale-level content validity index, averaging method $(\mathrm{S}-\mathrm{CVI} / \mathrm{Ave})=.85$; average proportion of items judged relevant across the ten experts $=.84$.

Table 6: Ratings on knowledge and clinical reasoning (disposition domain) scale by 10 experts

$\begin{array}{lcccccccccccc} & \text { Rater } & \text { Rater } & \text { Rater } & \text { Rater } & \text { Rater } & \text { Rater } & \text { Rater } & \text { Rater } & \text { Rater } & \text { Rater } & \text { Experts in Items } \\ \text { agreement CVI }\end{array}$

Note. I-CVI, item-level content validity index; scale-level content validity index, universal agreement method (S-CVI/UA) $=.89$; scale-level content validity index, averaging method $(\mathrm{S}-\mathrm{CVI} / \mathrm{Ave})=.89$; average proportion of items judged relevant across the ten experts $=.89$. 
CVI for the disposition domain (Table 6), item K3.2 was removed as it has zero I-CVI. A total 8 out of 9 items achieved acceptable universal agreement between experts $(\mathrm{S}-\mathrm{CVI} / \mathrm{UA}=0.89)$.

\section{Face Validity Index (FVI)}

Thirty candidates were selected from the expected respondents and their sociodemographic distribution were summarised in Table 7.

FVI of clarity among doctors (Table 8) was 0.97 and FVI clarity among paramedics (Table 9) was 0.87. FVI for comprehension of the doctors (Table 10) was 0.97 and comprehension of the paramedics (Table 11) was 0.83 .

Table 7: Sociodemographic characteristics of HCP participated in the face validation process

\begin{tabular}{lll}
\hline \multicolumn{1}{c}{ Variable } & n & (\%) \\
\hline Gender & & \\
Male & 13 & 43 \\
Female & 17 & 57 \\
Occupation & & \\
Medical Officer & 8 & 26.7 \\
House Officer & 7 & 23.3 \\
Medical Assistant & 7 & 23.3 \\
Nurse & 8 & 26.7 \\
\hline
\end{tabular}

Inter-rater reliability assessment using Intraclass Correlation Coefficient (ICC) was significant as shown in Table 12.

The standard setting for this tool consists of three phases which were: (a) marking scheme for each item generated and validated in knowledge component, (b) script concordance scoring for clinical reasoning component, and (c) cut off point scoring for differentiation of adequate/ inadequate knowledge or safe/unsafe clinical reasoning. At this stage, it just completed the first two out three phases. The allocated mark according to Table 13 focused specifically to the objective of each item. The answer scheme was referred to the blueprint of a specific domain.
Next part of standard setting scoring was for clinical reasoning component. The scoring distribution was shown in Figure 2, Figure 3 and Figure 4 for each domain. Two items were dropped which were CR5 (Figure 2, black line) and CR11 (Figure 3, grey line) due to poor discriminative value despite had significant score for CVI during the experts meeting and FVI value among users.

\section{DISCUSSION}

Acute asthma management in ED include the ability to diagnose, give appropriate treatment according to the clinical situation and plan for disposition. The purpose for developing K-CRAMED is to assess HCP's clinical knowledge and judgement based on certain clinical scenarios. All its items were developed based on two major guidelines (19), and thus providing strong evidence for its content validity. Apart from that, a well established technique such as the Delphi technique (15) used during development phase further strengthen its content validity. It is worthy to highlight that content validity is a prerequisite for any other forms of validity, thus should be given the highest priority during the development process of any new inventory (20). A new thing introduced by K-CRAMED is the clinical reasoning assessed by SCT that is based on the agreement test between experts and candidates (21). The idea is basically putting the candidate in the scenario and challenged them with input of additional clinical information (12). The candidates will give their response based on a Likert scale from least likely to more likely, and thus reflecting the reasoning towards a particular scenario. The key scoring of SCT was referred to the judgement of experts on each items based on the given scenario (22).

Several items were removed along the development process that primarily due to poor CVI. Item $\mathrm{K} 1.3$ was removed after meeting with the expert panel in view of allergy investigation is not part of the emergency department management. 
Table 8: Ratings on clarity of knowledge and clinical reasoning scale by 15 doctors

\begin{tabular}{llllllllllllllllll} 
& $\mathbf{R}$ & $\mathbf{R}$ & $\mathbf{R}$ & $\mathbf{R}$ & $\mathbf{R}$ & $\mathbf{R}$ & $\mathbf{R}$ & $\mathbf{R}$ & $\mathbf{R}$ & $\mathbf{R}$ & $\mathbf{R}$ & $\mathbf{R}$ & $\mathbf{R}$ & $\mathbf{R}$ & $\mathbf{R}$ & SUM & FVI \\
& $\mathbf{1}$ & $\mathbf{2}$ & $\mathbf{3}$ & $\mathbf{4}$ & $\mathbf{5}$ & $\mathbf{6}$ & $\mathbf{7}$ & $\mathbf{8}$ & $\mathbf{9}$ & $\mathbf{1 0}$ & $\mathbf{1 1}$ & $\mathbf{1 2}$ & $\mathbf{1 3}$ & $\mathbf{1 4}$ & $\mathbf{1 5}$ & \\
\hline Knowledge & & & & & & & & & & & & & & & & \\
Diagnosis & & & & & & & & & & & & & & & & \\
K1.1 & 4 & 4 & 4 & 4 & 4 & 4 & 4 & 4 & 4 & 4 & 4 & 4 & 4 & 4 & 4 & 60 & 1.00 \\
K1.2 & 4 & 4 & 4 & 4 & 4 & 4 & 4 & 4 & 4 & 4 & 4 & 4 & 4 & 4 & 4 & 60 & 1.00 \\
K1.4 & 4 & 4 & 4 & 4 & 4 & 4 & 4 & 4 & 4 & 4 & 3 & 4 & 4 & 4 & 3 & 58 & 0.97 \\
K1.5 & 4 & 4 & 4 & 4 & 4 & 4 & 4 & 4 & 4 & 4 & 4 & 4 & 4 & 4 & 4 & 60 & 1.00 \\
Treatment & & & & & & & & & & & & & & & & & \\
K2.1 & 4 & 4 & 4 & 4 & 4 & 4 & 4 & 4 & 4 & 4 & 4 & 4 & 4 & 4 & 4 & 60 & 1.00 \\
K2.2 & 4 & 4 & 4 & 4 & 4 & 4 & 4 & 4 & 4 & 3 & 4 & 4 & 4 & 4 & 4 & 59 & 0.98 \\
K2.3 & 4 & 4 & 4 & 4 & 4 & 4 & 4 & 4 & 4 & 4 & 4 & 3 & 4 & 4 & 4 & 59 & 0.98 \\
K2.4 & 4 & 4 & 4 & 4 & 4 & 4 & 4 & 4 & 3 & 3 & 3 & 4 & 4 & 4 & 4 & 57 & 0.95 \\
K2.6 & 4 & 4 & 4 & 4 & 4 & 4 & 4 & 4 & 4 & 4 & 3 & 3 & 4 & 4 & 4 & 58 & 0.97 \\
K2.7 & 4 & 4 & 4 & 4 & 4 & 4 & 4 & 4 & 3 & 4 & 4 & 4 & 4 & 4 & 4 & 59 & 0.98 \\
K2.8 & 4 & 4 & 4 & 4 & 4 & 4 & 4 & 4 & 4 & 3 & 3 & 3 & 4 & 4 & 4 & 57 & 0.95 \\
Disposition & & & & & & & & & & & & & & & & 4
\end{tabular}

\section{Clinical}

reasoning

Diagnosis

$\begin{array}{llllllllllllllllll}\text { CR1 } & 4 & 4 & 4 & 4 & 4 & 4 & 4 & 4 & 4 & 4 & 4 & 4 & 4 & 4 & 4 & 60 & 1.00 \\ \text { CR2 } & 4 & 4 & 4 & 4 & 4 & 4 & 4 & 4 & 4 & 3 & 4 & 4 & 4 & 4 & 4 & 59 & 0.98 \\ \text { CR3 } & 4 & 4 & 4 & 4 & 4 & 4 & 4 & 4 & 4 & 4 & 4 & 4 & 4 & 4 & 4 & 60 & 1.00 \\ \text { CR4 } & 4 & 4 & 4 & 4 & 4 & 4 & 4 & 4 & 4 & 3 & 3 & 3 & 4 & 4 & 3 & 56 & 0.93 \\ \text { CR5 } & 4 & 4 & 4 & 4 & 4 & 4 & 4 & 4 & 4 & 3 & 3 & 3 & 4 & 4 & 4 & 57 & 0.95 \\ \text { CR6 } & 4 & 4 & 4 & 4 & 4 & 4 & 4 & 4 & 4 & 3 & 3 & 3 & 4 & 3 & 4 & 56 & 0.93 \\ \text { CR7 } & 4 & 4 & 4 & 4 & 4 & 4 & 4 & 4 & 4 & 4 & 3 & 4 & 4 & 4 & 4 & 59 & 0.98 \\ \text { Treatment } & & & & & & & & & & & & & & & & & \\ \text { CR8 } & 4 & 4 & 4 & 4 & 4 & 4 & 4 & 4 & 3 & 3 & 3 & 4 & 4 & 4 & 4 & 57 & 0.95 \\ \text { CR9 } & 4 & 4 & 4 & 4 & 4 & 4 & 4 & 4 & 4 & 3 & 4 & 3 & 4 & 4 & 4 & 58 & 0.97 \\ \text { CR11 } & 4 & 4 & 4 & 4 & 4 & 4 & 4 & 4 & 4 & 3 & 4 & 3 & 4 & 3 & 4 & 57 & 0.95 \\ \text { CR12 } & 4 & 4 & 4 & 4 & 4 & 4 & 3 & 4 & 4 & 4 & 4 & 3 & 4 & 4 & 4 & 58 & 0.97 \\ \text { Disposition } & & & & & & & & & & & & & & & & & \\ \text { CR13 } & 4 & 4 & 4 & 4 & 4 & 4 & 3 & 4 & 4 & 4 & 3 & 3 & 4 & 4 & 4 & 57 & 0.95 \\ \text { CR14 } & 4 & 4 & 4 & 4 & 4 & 4 & 4 & 4 & 4 & 4 & 4 & 4 & 4 & 4 & 4 & 60 & 1.00 \\ \text { CR15 } & 4 & 4 & 4 & 4 & 4 & 4 & 4 & 4 & 4 & 3 & 3 & 3 & 4 & 3 & 4 & 56 & 0.93 \\ & & & & & & & & & & & & & & \text { FVI } & 0.97 & \end{array}$

Note. R: Rater; SUM: Summation; FVI: Face Validation Index; Scale 0-4 range: Difficult clarity - Easy clarity 
Table 9: Ratings on clarity of knowledge and clinical reasoning scale by 15 paramedics

\begin{tabular}{|c|c|c|c|c|c|c|c|c|c|c|c|c|c|c|c|c|c|}
\hline & $\begin{array}{l}R \\
1\end{array}$ & $\begin{array}{l}\mathbf{R} \\
2\end{array}$ & $\begin{array}{l}\mathbf{R} \\
\mathbf{3}\end{array}$ & $\begin{array}{l}R \\
4\end{array}$ & $\begin{array}{l}\mathbf{R} \\
\mathbf{5}\end{array}$ & $\begin{array}{l}R \\
6\end{array}$ & $\begin{array}{l}R \\
7\end{array}$ & $\begin{array}{l}R \\
8\end{array}$ & $\begin{array}{l}R \\
9\end{array}$ & $\begin{array}{c}R \\
10\end{array}$ & $\begin{array}{c}R \\
11\end{array}$ & $\begin{array}{c}R \\
12\end{array}$ & $\begin{array}{c}R \\
13\end{array}$ & $\begin{array}{c}R \\
14\end{array}$ & $\begin{array}{c}R \\
15\end{array}$ & SUM & FVI \\
\hline \multicolumn{18}{|c|}{ Knowledge } \\
\hline \multicolumn{18}{|c|}{ Diagnosis } \\
\hline K1.1 & 4 & 4 & 4 & 4 & 4 & 4 & 4 & 3 & 4 & 3 & 3 & 4 & 3 & 4 & 4 & 56 & 0.93 \\
\hline $\mathrm{K} 1.2$ & 4 & 4 & 3 & 4 & 4 & 3 & 3 & 3 & 4 & 3 & 3 & 3 & 3 & 3 & 4 & 51 & 0.85 \\
\hline K1.4 & 4 & 4 & 4 & 3 & 3 & 4 & 4 & 3 & 4 & 3 & 3 & 4 & 3 & 3 & 4 & 53 & 0.88 \\
\hline K1.5 & 4 & 4 & 4 & 3 & 4 & 4 & 4 & 3 & 4 & 4 & 3 & 4 & 3 & 3 & 4 & 55 & 0.92 \\
\hline \multicolumn{18}{|c|}{ Treatment } \\
\hline K2.1 & 4 & 4 & 4 & 4 & 3 & 4 & 3 & 3 & 4 & 4 & 3 & 4 & 3 & 4 & 4 & 55 & 0.92 \\
\hline $\mathrm{K} 2.2$ & 3 & 4 & 4 & 4 & 4 & 4 & 3 & 3 & 4 & 3 & 3 & 4 & 3 & 4 & 4 & 54 & 0.90 \\
\hline $\mathrm{K} 2.3$ & 4 & 4 & 4 & 3 & 3 & 4 & 3 & 3 & 4 & 3 & 3 & 3 & 3 & 3 & 4 & 51 & 0.85 \\
\hline $\mathrm{K} 2.4$ & 4 & 4 & 3 & 4 & 4 & 4 & 3 & 3 & 4 & 4 & 3 & 3 & 3 & 3 & 4 & 53 & 0.88 \\
\hline K2.6 & 4 & 4 & 4 & 3 & 3 & 4 & 3 & 2 & 4 & 2 & 3 & 3 & 3 & 3 & 4 & 49 & 0.82 \\
\hline K2.7 & 4 & 4 & 4 & 4 & 4 & 4 & 3 & 3 & 4 & 3 & 4 & 4 & 3 & 3 & 4 & 55 & 0.92 \\
\hline $\mathrm{K} 2.8$ & 3 & 4 & 4 & 4 & 4 & 4 & 3 & 3 & 4 & 3 & 3 & 4 & 3 & 3 & 4 & 53 & 0.88 \\
\hline \multicolumn{18}{|c|}{ Disposition } \\
\hline K3.1 & 4 & 4 & 4 & 4 & 4 & 3 & 4 & 3 & 4 & 3 & 3 & 4 & 3 & 3 & 4 & 54 & 0.90 \\
\hline K3.3 & 4 & 4 & 4 & 4 & 4 & 3 & 4 & 3 & 4 & 4 & 3 & 4 & 3 & 3 & 4 & 55 & 0.92 \\
\hline K3.4 & 3 & 4 & 3 & 3 & 3 & 3 & 3 & 2 & 4 & 3 & 3 & 3 & 3 & 3 & 4 & 47 & 0.78 \\
\hline K3.5 & 4 & 4 & 4 & 4 & 4 & 4 & 4 & 3 & 4 & 3 & 3 & 3 & 3 & 3 & 4 & 54 & 0.90 \\
\hline K3.6 & 4 & 4 & 4 & 4 & 4 & 4 & 4 & 3 & 4 & 3 & 3 & 4 & 3 & 3 & 4 & 55 & 0.92 \\
\hline \multicolumn{18}{|c|}{$\begin{array}{l}\text { Clinical } \\
\text { reasoning }\end{array}$} \\
\hline \multicolumn{18}{|c|}{ Diagnosis } \\
\hline CR1 & 4 & 4 & 4 & 4 & 4 & 3 & 3 & 2 & 4 & 3 & 3 & 3 & 3 & 3 & 4 & 51 & 0.85 \\
\hline CR2 & 4 & 4 & 4 & 3 & 3 & 3 & 3 & 2 & 4 & 3 & 3 & 3 & 3 & 3 & 4 & 49 & 0.82 \\
\hline CR3 & 4 & 4 & 4 & 4 & 4 & 3 & 3 & 3 & 4 & 3 & 4 & 3 & 3 & 3 & 4 & 53 & 0.88 \\
\hline CR4 & 4 & 4 & 4 & 4 & 4 & 3 & 3 & 2 & 4 & 3 & 4 & 3 & 3 & 3 & 4 & 52 & 0.87 \\
\hline CR5 & 4 & 4 & 4 & 4 & 4 & 3 & 3 & 3 & 4 & 3 & 4 & 4 & 3 & 3 & 4 & 54 & 0.90 \\
\hline CR6 & 4 & 4 & 4 & 4 & 4 & 3 & 3 & 3 & 4 & 3 & 3 & 4 & 3 & 3 & 4 & 53 & 0.88 \\
\hline CR7 & 4 & 4 & 4 & 3 & 3 & 3 & 3 & 3 & 4 & 3 & 3 & 4 & 3 & 3 & 4 & 51 & 0.85 \\
\hline \multicolumn{18}{|c|}{ Treatment } \\
\hline CR8 & 4 & 4 & 4 & 4 & 3 & 3 & 3 & 3 & 4 & 3 & 3 & 4 & 3 & 2 & 4 & 51 & 0.85 \\
\hline CR9 & 3 & 4 & 4 & 4 & 4 & 3 & 3 & 3 & 4 & 3 & 3 & 4 & 3 & 3 & 4 & 52 & 0.87 \\
\hline CR11 & 4 & 4 & 4 & 4 & 4 & 3 & 3 & 2 & 4 & 3 & 3 & 4 & 3 & 3 & 4 & 52 & 0.87 \\
\hline CR12 & 4 & 4 & 4 & 3 & 3 & 3 & 3 & 2 & 4 & 3 & 3 & 3 & 3 & 3 & 4 & 49 & 0.82 \\
\hline \multicolumn{18}{|c|}{ Disposition } \\
\hline CR13 & 4 & 4 & 4 & 3 & 3 & 3 & 3 & 2 & 4 & 3 & 3 & 3 & 3 & 3 & 4 & 49 & 0.82 \\
\hline CR14 & 4 & 4 & 4 & 3 & 4 & 3 & 3 & 3 & 4 & 3 & 3 & 3 & 3 & 3 & 4 & 51 & 0.85 \\
\hline \multirow[t]{2}{*}{ CR15 } & 4 & 4 & 4 & 3 & 4 & 3 & 3 & 3 & 4 & 3 & 3 & 4 & 3 & 3 & 4 & 52 & 0.87 \\
\hline & & & & & & & & & & & & & & $\begin{array}{c}\text { FVI } \\
\text { Average }\end{array}$ & 0.87 & & \\
\hline
\end{tabular}

Note. R: Rater; SUM: Summation; FVI: Face Validation Index; Scale 0-4 range: Difficult clarity - Easy clarity 
Table 10: Ratings on comprehensibility of knowledge and clinical reasoning scale by 15 doctors

\begin{tabular}{|c|c|c|c|c|c|c|c|c|c|c|c|c|c|c|c|c|c|}
\hline & $\begin{array}{c}\mathbf{R} \\
\mathbf{1}\end{array}$ & $\begin{array}{l}\mathbf{R} \\
\mathbf{2}\end{array}$ & $\begin{array}{l}\mathbf{R} \\
\mathbf{3}\end{array}$ & $\begin{array}{l}R \\
4\end{array}$ & $\begin{array}{l}R \\
5\end{array}$ & $\begin{array}{l}R \\
6\end{array}$ & $\begin{array}{l}R \\
7\end{array}$ & $\begin{array}{l}\mathbf{R} \\
\mathbf{8}\end{array}$ & $\begin{array}{l}\mathbf{R} \\
\mathbf{9}\end{array}$ & $\begin{array}{c}R \\
10\end{array}$ & $\begin{array}{c}R \\
11\end{array}$ & $\begin{array}{c}R \\
12\end{array}$ & $\begin{array}{c}R \\
13\end{array}$ & $\begin{array}{c}R \\
14\end{array}$ & $\begin{array}{c}R \\
15\end{array}$ & SUM & FVI \\
\hline \multicolumn{18}{|c|}{ Knowledge } \\
\hline \multicolumn{18}{|c|}{ Diagnosis } \\
\hline K1.1 & 4 & 4 & 4 & 4 & 4 & 4 & 4 & 4 & 4 & 4 & 4 & 4 & 4 & 4 & 4 & 60 & 1.00 \\
\hline $\mathrm{K} 1.2$ & 4 & 4 & 4 & 4 & 4 & 4 & 4 & 4 & 4 & 4 & 4 & 4 & 4 & 4 & 4 & 60 & 1.00 \\
\hline K1.4 & 4 & 4 & 4 & 4 & 4 & 4 & 4 & 4 & 4 & 3 & 3 & 4 & 4 & 4 & 3 & 57 & 0.95 \\
\hline K1.5 & 4 & 4 & 4 & 4 & 4 & 4 & 4 & 4 & 4 & 4 & 4 & 4 & 4 & 4 & 4 & 60 & 1.00 \\
\hline \multicolumn{18}{|c|}{ Treatment } \\
\hline K2.1 & 4 & 4 & 4 & 4 & 4 & 4 & 4 & 4 & 4 & 4 & 3 & 4 & 3 & 4 & 4 & 58 & 0.97 \\
\hline $\mathrm{K} 2.2$ & 4 & 4 & 4 & 4 & 4 & 4 & 4 & 4 & 4 & 3 & 4 & 4 & 4 & 4 & 4 & 59 & 0.98 \\
\hline K2.3 & 4 & 4 & 4 & 4 & 4 & 4 & 4 & 4 & 4 & 4 & 4 & 4 & 4 & 4 & 4 & 60 & 1.00 \\
\hline K2.4 & 4 & 4 & 4 & 4 & 4 & 4 & 4 & 4 & 4 & 3 & 4 & 4 & 4 & 4 & 4 & 59 & 0.98 \\
\hline K2.6 & 4 & 4 & 4 & 4 & 4 & 4 & 4 & 4 & 4 & 4 & 3 & 4 & 4 & 4 & 4 & 59 & 0.98 \\
\hline K2.7 & 4 & 4 & 4 & 4 & 4 & 4 & 4 & 4 & 4 & 4 & 4 & 4 & 4 & 4 & 4 & 60 & 1.00 \\
\hline K2.8 & 4 & 4 & 4 & 4 & 4 & 4 & 4 & 4 & 4 & 3 & 3 & 4 & 4 & 4 & 4 & 58 & 0.97 \\
\hline \multicolumn{18}{|c|}{ Disposition } \\
\hline K3.1 & 3 & 3 & 4 & 3 & 4 & 4 & 4 & 3 & 3 & 3 & 3 & 4 & 4 & 3 & 3 & 51 & 0.85 \\
\hline K3.3 & 4 & 4 & 4 & 4 & 4 & 4 & 3 & 4 & 4 & 4 & 4 & 4 & 4 & 4 & 4 & 59 & 0.98 \\
\hline K3.4 & 4 & 4 & 4 & 4 & 4 & 4 & 4 & 4 & 4 & 3 & 4 & 3 & 3 & 3 & 4 & 56 & 0.93 \\
\hline K3.5 & 4 & 4 & 4 & 4 & 4 & 3 & 4 & 4 & 4 & 3 & 3 & 4 & 4 & 4 & 4 & 57 & 0.95 \\
\hline K3.6 & 4 & 4 & 4 & 4 & 4 & 4 & 4 & 4 & 4 & 3 & 3 & 3 & 4 & 4 & 4 & 57 & 0.95 \\
\hline
\end{tabular}

\section{Clinical}

reasoning

Diagnosis

\begin{tabular}{|c|c|c|c|c|c|c|c|c|c|c|c|c|c|c|c|c|c|}
\hline CR1 & 4 & 4 & 4 & 4 & 4 & 4 & 4 & 4 & 4 & 4 & 4 & 4 & 4 & 4 & 4 & 60 & 1.00 \\
\hline CR2 & 4 & 4 & 4 & 4 & 4 & 4 & 4 & 4 & 4 & 4 & 4 & 4 & 4 & 4 & 4 & 60 & 1.00 \\
\hline CR3 & 4 & 4 & 4 & 4 & 4 & 4 & 4 & 4 & 4 & 4 & 4 & 4 & 4 & 4 & 4 & 60 & 1.00 \\
\hline CR4 & 4 & 4 & 4 & 4 & 4 & 4 & 4 & 4 & 4 & 3 & 3 & 4 & 4 & 4 & 3 & 57 & 0.95 \\
\hline CR5 & 4 & 4 & 4 & 4 & 4 & 4 & 4 & 4 & 3 & 4 & 4 & 4 & 4 & 4 & 4 & 59 & 0.98 \\
\hline CR6 & 4 & 4 & 4 & 4 & 4 & 4 & 4 & 4 & 3 & 4 & 4 & 4 & 4 & 3 & 4 & 58 & 0.97 \\
\hline CR7 & 4 & 4 & 4 & 4 & 4 & 4 & 4 & 4 & 4 & 4 & 3 & 4 & 4 & 4 & 4 & 59 & 0.98 \\
\hline \multicolumn{18}{|c|}{ Treatment } \\
\hline CR8 & 4 & 4 & 4 & 4 & 4 & 3 & 4 & 4 & 3 & 3 & 3 & 3 & 4 & 4 & 4 & 55 & 0.92 \\
\hline CR9 & 4 & 4 & 4 & 4 & 4 & 4 & 4 & 4 & 4 & 3 & 4 & 3 & 4 & 4 & 4 & 58 & 0.97 \\
\hline CR11 & 4 & 4 & 4 & 4 & 4 & 4 & 4 & 4 & 4 & 4 & 4 & 4 & 4 & 3 & 4 & 59 & 0.98 \\
\hline CR12 & 4 & 4 & 4 & 4 & 4 & 4 & 4 & 4 & 4 & 3 & 4 & 3 & 4 & 4 & 4 & 58 & 0.97 \\
\hline \multicolumn{18}{|c|}{ Disposition } \\
\hline CR13 & 4 & 4 & 4 & 4 & 4 & 3 & 3 & 4 & 4 & 4 & 3 & 3 & 4 & 4 & 4 & 56 & 0.93 \\
\hline CR14 & 4 & 4 & 4 & 4 & 4 & 4 & 4 & 4 & 4 & 4 & 4 & 4 & 4 & 4 & 4 & 60 & 1.00 \\
\hline \multirow[t]{2}{*}{ CR15 } & 4 & 4 & 4 & 4 & 4 & 4 & 4 & 4 & 4 & 4 & 3 & 3 & 4 & 3 & 4 & 57 & 0.95 \\
\hline & & & & & & & & & & & & & & $\begin{array}{c}\text { FVI } \\
\text { Average }\end{array}$ & 0.97 & & \\
\hline
\end{tabular}

Note. R: Rater; SUM: Summation; FVI: Face Validation Index; Scale 0-4 range: Difficult comprehensibility - Easy comprehensibility 
Table 11: Ratings on comprehensibility of knowledge and clinical reasoning scale by 15 paramedics

$\begin{array}{llllllllllllllllll} & \mathbf{R} & \mathbf{R} & \mathbf{R} & \mathbf{R} & \mathbf{R} & \mathbf{R} & \mathbf{R} & \mathbf{R} & \mathbf{R} & \mathbf{R} & \mathbf{R} & \mathbf{R} & \mathbf{R} & \mathbf{R} & \mathbf{R} & \text { SUM } & \text { FVI } \\ & \mathbf{1} & \mathbf{2} & \mathbf{3} & \mathbf{4} & \mathbf{5} & \mathbf{6} & \mathbf{7} & \mathbf{8} & \mathbf{9} & \mathbf{1 0} & \mathbf{1 1} & \mathbf{1 2} & \mathbf{1 3} & \mathbf{1 4} & \mathbf{1 5} & \\ \text { Knowledge } & & & & & & & & & & & & & & & & \\ \text { Diagnosis } & & & & & & & & & & & & & & & & \\ \text { K1.1 } & 4 & 4 & 4 & 3 & 4 & 4 & 4 & 3 & 4 & 3 & 3 & 3 & 3 & 4 & 4 & 54 & 0.90 \\ \text { K1.2 } & 4 & 3 & 4 & 3 & 4 & 3 & 3 & 3 & 4 & 3 & 3 & 3 & 4 & 3 & 4 & 51 & 0.85 \\ \text { K1.4 } & 4 & 3 & 4 & 3 & 4 & 3 & 4 & 3 & 4 & 3 & 2 & 3 & 4 & 3 & 4 & 51 & 0.85 \\ \text { K1.5 } & 4 & 4 & 4 & 3 & 4 & 3 & 4 & 3 & 4 & 4 & 3 & 3 & 4 & 3 & 4 & 54 & 0.90 \\ \text { Treatment } & & & & & & & & & & & & & & & & & \\ \text { K2.1 } & 4 & 4 & 4 & 3 & 3 & 3 & 3 & 3 & 4 & 4 & 3 & 3 & 3 & 3 & 4 & 51 & 0.85 \\ \text { K2.2 } & 3 & 4 & 4 & 3 & 3 & 3 & 3 & 3 & 4 & 3 & 3 & 3 & 3 & 3 & 4 & 49 & 0.82 \\ \text { K2.3 } & 4 & 4 & 4 & 3 & 3 & 3 & 3 & 3 & 4 & 3 & 3 & 3 & 3 & 3 & 4 & 50 & 0.83 \\ \text { K2.4 } & 4 & 4 & 4 & 3 & 4 & 3 & 3 & 3 & 4 & 4 & 3 & 4 & 4 & 3 & 4 & 54 & 0.90 \\ \text { K2.6 } & 4 & 4 & 4 & 3 & 3 & 3 & 3 & 3 & 4 & 2 & 3 & 3 & 3 & 3 & 4 & 49 & 0.82 \\ \text { K2.7 } & 4 & 4 & 4 & 3 & 4 & 3 & 3 & 3 & 4 & 3 & 4 & 3 & 3 & 3 & 4 & 52 & 0.87 \\ \text { K2.8 } & 3 & 4 & 4 & 3 & 4 & 3 & 3 & 3 & 4 & 3 & 3 & 3 & 3 & 3 & 4 & 50 & 0.83 \\ \text { Disposition } & & & & & & & & & & & & & & & & 4 & 47 \\ \text { K3.1 } & 3 & 3 & 3 & 3 & 4 & 3 & 3 & 3 & 3 & 3 & 3 & 3 & 3 & 3 & 4 & 47 \\ \text { K3.3 } & 4 & 3 & 4 & 3 & 4 & 3 & 4 & 3 & 4 & 4 & 3 & 4 & 3 & 3 & 4 & 53 & 0.88 \\ \text { K3.4 } & 3 & 3 & 3 & 3 & 3 & 3 & 3 & 2 & 4 & 3 & 3 & 2 & 3 & 3 & 4 & 45 & 0.75 \\ \text { K3.5 } & 4 & 4 & 3 & 3 & 4 & 3 & 4 & 2 & 4 & 3 & 2 & 2 & 3 & 2 & 4 & 47 & 0.78 \\ \text { K3.6 } & 4 & 4 & 4 & 3 & 4 & 3 & 4 & 3 & 4 & 3 & 3 & 3 & 3 & 3 & 4 & 52 & 0.87\end{array}$

\section{Clinical}

reasoning

Diagnosis

$\begin{array}{llllllllllllllllll}\text { CR1 } & 4 & 3 & 4 & 3 & 4 & 3 & 3 & 2 & 4 & 3 & 3 & 4 & 3 & 3 & 4 & 50 & 0.83 \\ \text { CR2 } & 4 & 3 & 3 & 3 & 3 & 3 & 3 & 2 & 4 & 3 & 3 & 4 & 3 & 3 & 4 & 48 & 0.80 \\ \text { CR3 } & 4 & 3 & 4 & 3 & 4 & 3 & 3 & 3 & 4 & 3 & 4 & 4 & 3 & 3 & 4 & 52 & 0.87 \\ \text { CR4 } & 4 & 3 & 4 & 3 & 4 & 3 & 3 & 2 & 4 & 3 & 4 & 4 & 3 & 3 & 4 & 51 & 0.85 \\ \text { CR5 } & 4 & 3 & 4 & 3 & 4 & 3 & 3 & 3 & 4 & 3 & 4 & 4 & 3 & 3 & 4 & 52 & 0.87 \\ \text { CR6 } & 4 & 3 & 4 & 3 & 4 & 3 & 3 & 3 & 4 & 3 & 3 & 4 & 3 & 3 & 4 & 51 & 0.85 \\ \text { CR7 } & 4 & 3 & 3 & 3 & 3 & 3 & 3 & 3 & 4 & 3 & 3 & 3 & 2 & 3 & 4 & 47 & 0.78\end{array}$

Treatment

\begin{tabular}{|c|c|c|c|c|c|c|c|c|c|c|c|c|c|c|c|c|c|}
\hline CR8 & 4 & 3 & 4 & 3 & 3 & 3 & 3 & 3 & 4 & 3 & 3 & 3 & 2 & 2 & 4 & 47 & 0.78 \\
\hline CR9 & 3 & 3 & 4 & 3 & 4 & 3 & 3 & 3 & 4 & 3 & 3 & 3 & 3 & 3 & 4 & 49 & 0.82 \\
\hline CR11 & 4 & 3 & 4 & 3 & 4 & 3 & 3 & 2 & 4 & 2 & 3 & 3 & 3 & 3 & 4 & 48 & 0.80 \\
\hline CR12 & 4 & 3 & 4 & 3 & 3 & 3 & 3 & 2 & 4 & 3 & 3 & 2 & 3 & 3 & 4 & 47 & 0.78 \\
\hline \multicolumn{18}{|c|}{ Disposition } \\
\hline CR13 & 4 & 3 & 4 & 3 & 3 & 3 & 3 & 2 & 4 & 3 & 3 & 3 & 3 & 3 & 4 & 48 & 0.80 \\
\hline CR14 & 4 & 3 & 4 & 3 & 4 & 3 & 3 & 3 & 4 & 3 & 3 & 3 & 3 & 3 & 4 & 50 & 0.83 \\
\hline \multirow[t]{2}{*}{ CR15 } & 4 & 3 & 4 & 3 & 4 & 3 & 3 & 3 & 4 & 3 & 3 & 3 & 3 & 3 & 4 & 50 & 0.83 \\
\hline & & & & & & & & & & & & & & $\begin{array}{c}\text { FVI } \\
\text { Average }\end{array}$ & 0.83 & & \\
\hline
\end{tabular}

Note. R: Rater; SUM: Summation; FVI: Face Validation Index; Scale 0-4 range: Difficult comprehensibility - Easy comprehensibility 
Table 12: Inter-rater Agreement for the Expert Panel $(\mathrm{N}=10)$

\begin{tabular}{cccc}
\hline Variable & $\begin{array}{c}\text { *Intraclass Correlation Coefficient } \\
(\mathbf{9 5 \%} \text { Cl; Lower, Upper) }\end{array}$ & F Test & P value \\
\hline Expert judgement & $0.989(0.982,0.994)$ & 114.274 & $<0.001$ \\
\hline \multicolumn{4}{c}{ Average measure } \\
\end{tabular}

Table 13: Recommended mark for each item in knowledge component

\begin{tabular}{cc}
\hline Item & Recommended mark \\
\hline K1.1 & 4 \\
K1.2 & 1 \\
K1.4 & 2 \\
K1.5 & 3 \\
K2.1 & 1 \\
K2.2 & 1 \\
K2.3 & 5 \\
K2.4 & 3 \\
K2.6 & 1 \\
K2.7 & 2 \\
K2.8 & 2 \\
K3.1 & 3 \\
K3.3 & 2 \\
K3.4 & 1 \\
K3.5 & 1 \\
K3.6 & 3 \\
\hline
\end{tabular}

Conversely, knowing the allergy history is important and educating the patient with knowledge of possible allergen may help to improve the management of asthma care.

Item K2.5 was considered not relevant by the expert panel as knowing the preparation of Combivent is not mandatory. The knowledge on how to prepare the medication in the form of a combination between salbutamol and Ipratropium bromide is much more important. Combivent is just a ready-made preparation by pharmaceutical company in which the dosage of its preparation should be available on each ampoule. Remembering this readymade preparation will not contribute in improvement of patient care.

Last item dropped was K3.2. In an emergency setting, knowing the diagnosis of allergy rhinitis and eczema would facilitate in increasing the likelihood diagnosis of asthma. However, the arrangement of the follow up care for those two problems

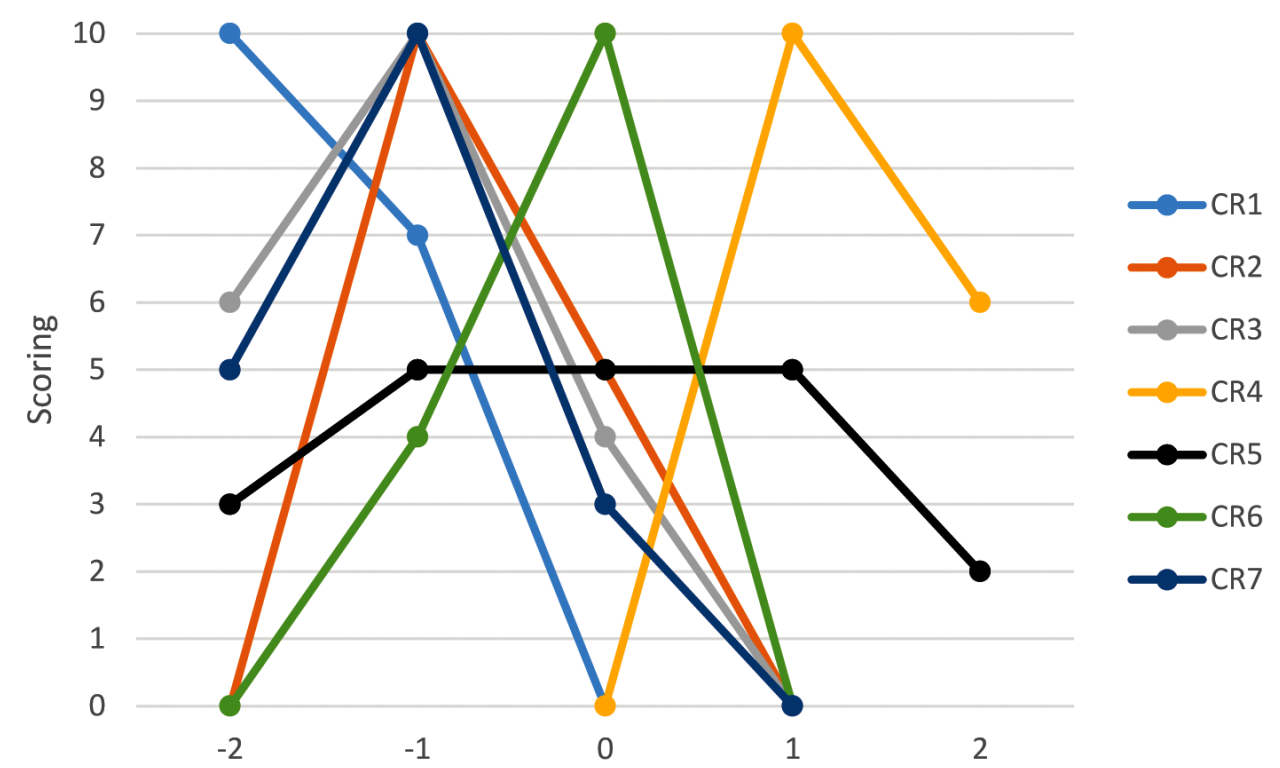

Figure 2: Mark distribution for clinical reasoning (Domain of diagnosis) $(-2$ to +2$)$. 


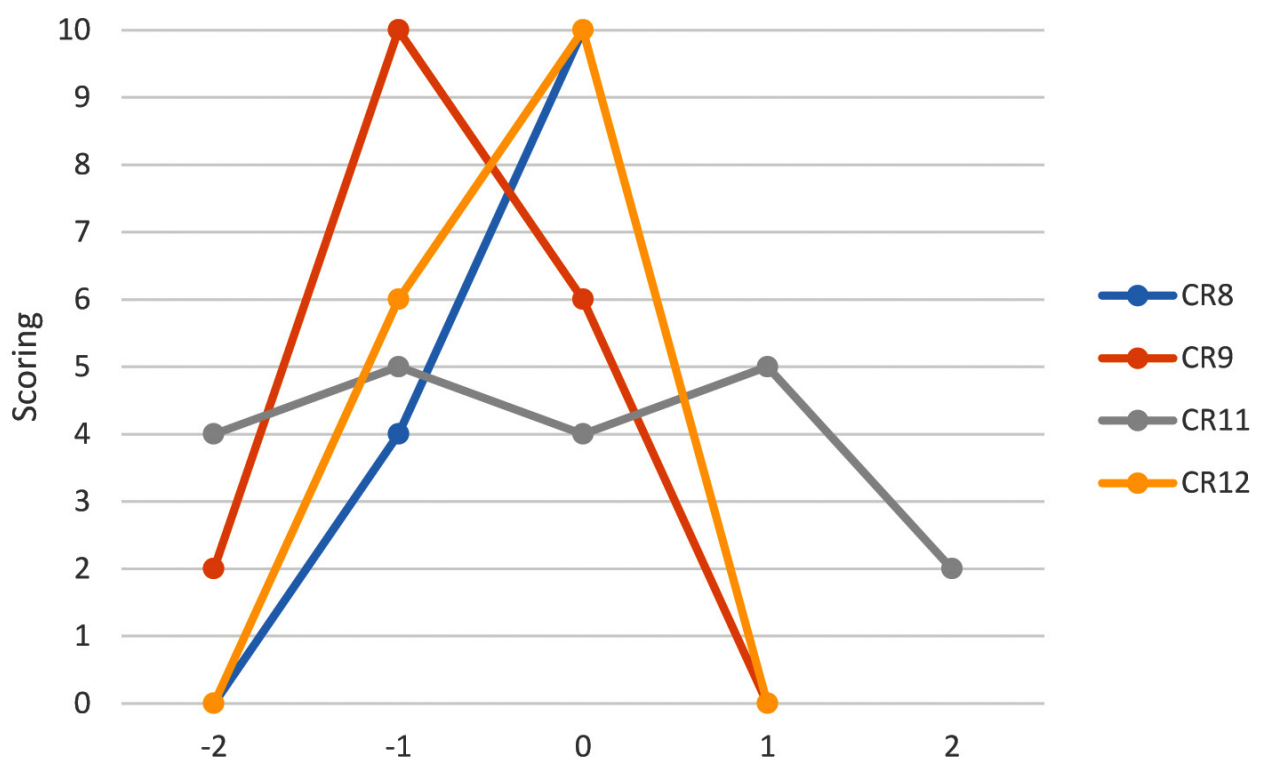

Figure 3: Mark distribution for clinical reasoning (Domain of treatment) $(-2$ to +2$)$.

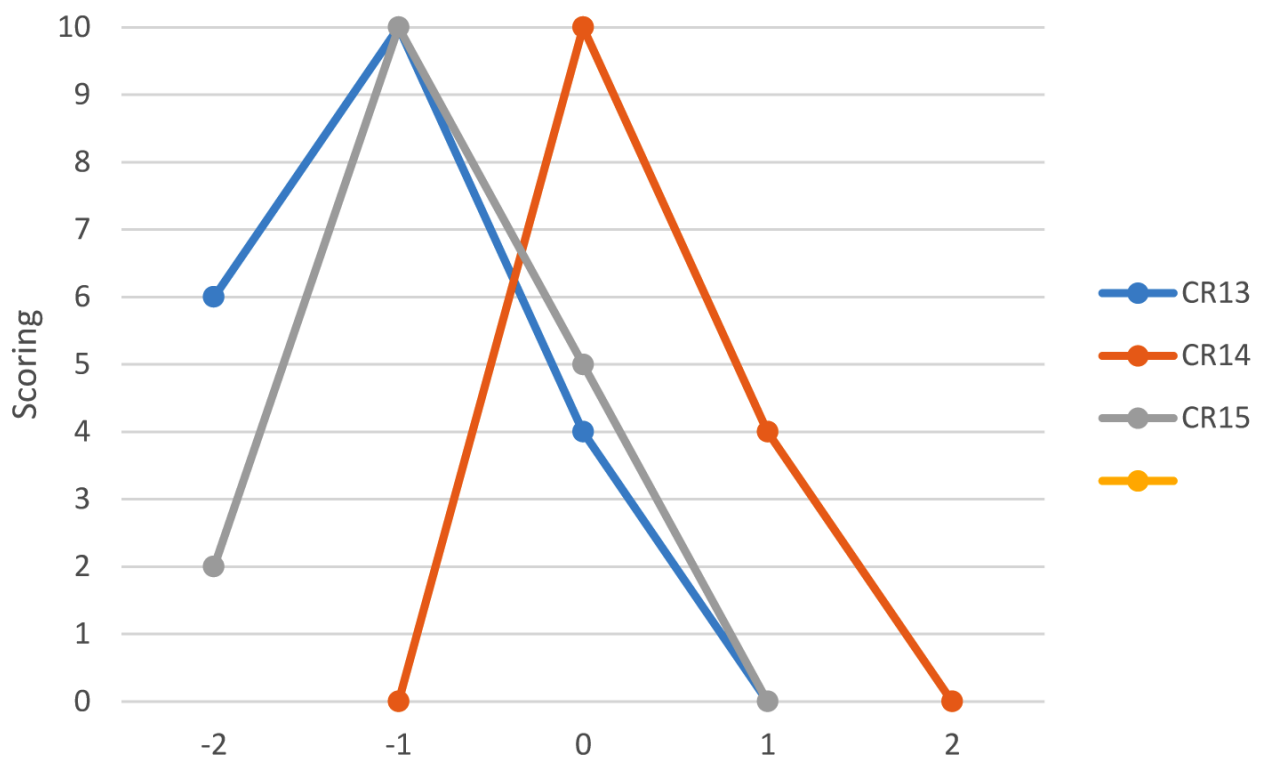

Figure 4: Mark distribution for clinical reasoning (Domain of disposition) ( -2 to +2$)$.

needs a proper consensus of agreement in each centre. So far, there was no concrete conclusion of compulsory review in each otorhinolaryngology clinic or dermatology clinic for the asthma exacerbation patient that been discharged from emergency department.

Item CR10 was about challenging the idea of giving intravenous Salbutamol, however all experts agreed that with current evidence, the usage of intravenous form should not be advocated. Besides, this tool is not only for tertiary care centre, but also for primary and secondary care centre. By giving the idea of intravenous usage of Salbutamol, it may lead to unnecessary misleading spread of information that it is generally accepted. Despite removal of the items, the main objective remains the same as this tool focuses on assessing clinical knowledge and reasoning in managing asthma exacerbation in emergency department. The final numbers of items at 
the end of development stage was 28 items. The 28 items were inside the following domains: 10 items in the diagnosis domain, 10 items in the treatment domain and 8 items in the disposition domain. The CVI of the final items was more than 0.83, indicating an acceptable level of content validity (16-18).

Pertaining to the response process as represented by FVI, the 28 items scored a high level of face validity in term of its clarity and comprehensibility, indicating a good response process (23). However, there was a difference between paramedics and doctors. One of concerns highlighted was the length of statement negatively influence the clarity and comprehensibility. The second concern was the language barriers due to the items were constructed in English language, thus make it difficult to be understood by paramedics.

To address these concerns, two major adjustments were made:

1. For clinical reasoning component, additional information were added in the scenario and the clinical judgement were colour-coded;

2. For the knowledge component, ambiguous terms were highlighted, reworded and rephrased to facilitate the clarity and comprehensibility of items.

Reassessment of the specific section showed better scores for clarity and comprehensibility, and thus providing a strong response process evidence through evaluation of the K-CRAMED items' appearance (10).

The final part was setting the key scoring for the knowledge via supplied type questions and the clinical reasoning via SCT. Despite having high CVI, two items (CR5 and CR11) were found to have poor discriminant as the scores given by the experts varies and could not reach consensus on the scoring. Future study should investigate on the cut-off point to differentiate between good and poor management of acute asthmatic patients. Standard setting of benchmark score for each specific group of HCP like medical doctors, houseman doctors, and paramedics should be estimated differently. In addition, this new tool is an examination setting, thus developing benchmark score might be beneficial to facilitate researchers in identifying poor candidate during the assessment. Apart from that, this tool is best kept by the researcher only, unless population assessment plan is to be conducted (to prevent from bias assessment). This tool might also be able to help researchers in assessing pre and post intervention in educating HCP regarding acute asthma exacerbation management.

\section{CONCLUSION}

This study has shown K-CRAMED is a valid tool to assess knowledge and clinical reasoning of $\mathrm{HCP}$ in managing acute asthmatic patients. However, further assessment is required to verify its psychometric credentials in different settings.

\section{REFERENCES}

1. Masoli M, Fabian D, Holt S, Beasley R. The global burden of asthma: executive summary of the GINA dissemination committee report. Allergy. 2004;59(5):469-78. https:// doi.org/10.1111/j.1398-9995.2004.00526.x.

2. Marks G, Pearce N, Strachan D, Asher I. The burden of disease due to asthma. In: Global asthma report 2014. Aukland, New Zealand: Global Asthma Network; 2014. p. 16-21.

3. David S, Elizabeth L, Neil P, Guy M. Asthma mortality. In: Global asthma report 2014. Aukland, New Zealand: Global Asthma Network; 2014. p. 28-32. 
4. Mohsen S, J Mark FitzGerald. The economic burden of asthma. In: Global asthma report 2014. Aukland, New Zealand: Global Asthma Network; 2014. p. 36-38.

5. Ting S. Multi-colored simplified asthma guideline reminder. Clinical Reviews in Allergy \& Immunology. 2004;27(2):133-45. https://doi.org/10.1385/CRIAI:27:2:133.

6. Almutawa FN, Al-Mutairy G, Al-Arada $\mathrm{N}$, Kamel MI. Perception of primary care physicians about guidelines of bronchial asthma. Alexandria Journal of Medicine. 2014;50(1):17-24. https://doi.org/10.1016/j. ajme.2013.05.002.

7. Lagerlov $P$, Veninga C, Muskova M, Hummers-Pradier E, Lundborg CS, Andrew M, Haaijer-Ruskamp FM. Asthma management in five European countries: doctors' knowledge, attitudes and prescribing behaviour. Drug Education Project (DEP) group. European Respiratory Journal. 2000;15(1):25-9. https://doi.org/10.1183/09 031936.00 .15102500 .

8. Wahabi HA, Alziedan RA. Reasons behind non-adherence of healthcare practitioners to pediatric asthma guidelines in an emergency department in Saudi Arabia. BMC Health Services Research. 2012;12(1):226. https:// doi.org/10.1186/1472-6963-12-226.

9. Sun $\mathrm{YH}$, Eun BW, Sim S-Y, Cho K-H, Ryoo E, Choi DY, Son DW, Tchah H, Jeon IS. Poor adherence and reasons for nonadherence to the asthma guidelines among pediatricians in Korea. Asian Pacific Journal of Allergy and Immunology. 2010;28(2-3):147-54.

10. Cook DA, Beckman TJ. Current concepts in validity and reliability for psychometric instruments: theory and application. The American Journal of Medicine. 2006;119(2):166.e7-e16.

11. Ballou J. Open-ended question. Encyclopedia of Survey Research Methods. 2008;43:548-50.
12. Charlin B, Roy L, Brailovsky C, Goulet F, van der Vleuten C. The script concordance test: a tool to assess the reflective clinician. Teaching and Learning in Medicine. 2000;12(4):189-95. https://doi.org/10.1207/ S15328015TLM1204_5.

13. Global initiative for asthma (GINA). Global strategy for asthma management and prevention. 2015. Available from: http:// ginasthma.org/wp-content/uploads/2016/01/ GINA_Pocket_2015.pdf

14. British Thoracic Society (BTS) SIGNS. British guideline on the management of asthma: a national clinical guideline. 2014. Available from: https://www.britthoracic.org.uk/document-library/clinicalinformation/asthma/btssign-asthmaguideline-2014/

15. Hsu C-C, Stanford BA. The delphi technique: making sense of consensus. Practical Assessment, Research \& Evaluation. 2007;12(10):1-8.

16. Polit DF, Beck CT. The content validity index: are you sure you know what's being reported? Critique and recommendations. Research in Nursing and Health. 2006;29(5):489-97. https://doi.org/10.1002/ nur.20147.

17. Polit D, Beck T, Owen S. Focus on research methods is the CVI an acceptable indicator of content validity. Res Nurs Health. 2007;30:459-67. https://doi.org/10.1002/ nur.20199.

18. Davis LL. Instrument review: getting the most from a panel of experts. Applied Nursing Research. 1992;5(4):194-7. https:// doi.org/10.1016/S0897-1897(05)80008-4.

19. Philippa E, Innes A, Karen B, Guy M, Asma ES, Eamon E. Asthma management guidelines. In: Global asthma report 2014. Aukland, New Zealand: Global Asthma Network; 2014. p. 48-51. 
20. Zamanzadeh V, Ghahramanian A, Rassouli M, Abbaszadeh A, Alavi-Majd H, Nikanfar A-R. Design and implementation content validity study: development of an instrument for measuring patient-centered communication. Journal of Caring Sciences. 2015;4(2):165. https://doi.org/10.15171/ jcs.2015.017.

21. Lubarsky S, Dory V, Duggan P, Gagnon R, Charlin B. Script concordance testing: from theory to practice. AMEE Guide No. 75. Medical Teacher. 2013;35(3):184-93. https://doi.org/10.3109/0142159X.2013. 760036.
22. Fournier JP, Demeester A, Charlin B. Script concordance tests: guidelines for construction. BMC Medical Informatics and Decision Making. 2008;8(1):18. https://doi. org/10.1186/1472-6947-8-18.

23. DeVon HA, Block ME, Moyle-Wright P, Ernst DM, Hayden SJ, Lazzara DJ, Savoy SM, Kostas-Polston E. A psychometric toolbox for testing validity and reliability. Journal of Nursing Scholarship. 2007;39(2):155-64. https://doi.org/10.1111/ j.1547-5069.2007.00161.x.w 